Audiology

Neurotology
Audiol Neurotol 2014;19:400-411

DOI: $10.1159 / 000365273$
Received: July 22, 2013

Accepted after revision: June 16, 2014

Published online: November 7, 2014

\title{
Clinical Evaluation of an Image-Guided Cochlear Implant Programming Strategy
}

\author{
Jack H. Noble ${ }^{a}$ René H. Gifford ${ }^{b}$ Andrea J. Hedley-Williams ${ }^{b}$ \\ Benoit M. Dawant ${ }^{\mathrm{a}}$ Robert F. Labadie ${ }^{\mathrm{c}}$

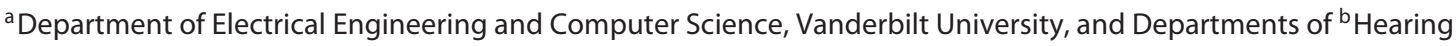 \\ and Speech Science and ' Otolaryngology - Head and Neck Surgery, Vanderbilt University Medical Center, \\ Nashville, Tenn., USA
}

\section{Key Words}

Cochlear implant · Stimulation overlap · Channel

interaction $\cdot$ Customized programming

\begin{abstract}
The cochlear implant $(\mathrm{Cl})$ has been labeled the most successful neural prosthesis. Despite this success, a significant number of $\mathrm{Cl}$ recipients experience poor speech understanding, and, even among the best performers, restoration to normal auditory fidelity is rare. While significant research efforts have been devoted to improving stimulation strategies, few developments have led to significant hearing improvement over the past two decades. We have recently introduced image processing techniques that open a new direction for advancement in this field by making it possible, for the first time, to determine the position of implanted $\mathrm{Cl}$ electrodes relative to the nerves they stimulate using computed tomography images. In this article, we present results of an image-guided, patient-customized approach to stimulation that utilizes the electrode position information our image processing techniques provide. This approach allows us to identify electrodes that cause overlapping stimulation patterns and to deactivate them from a patient's map. This individualized mapping strategy yields significant improvement in speech understanding in both quiet and noise as well as
\end{abstract}

improved spectral resolution in the 68 adult $\mathrm{Cl}$ recipients studied to date. Our results indicate that image guidance can improve hearing outcomes for many existing $\mathrm{Cl}$ recipients without requiring additional surgery or the use of 'experimental' stimulation strategies, hardware or software.

(c) 2014 S. Karger AG, Basel

\section{Introduction}

Cochlear implants (CIs) are surgically implanted neural prosthetic devices used to treat severe-to-profound hearing loss [NIDCD, 2011]. To date, the CI has arguably been the most successful neural prosthesis. CIs use implanted electrodes to stimulate spiral ganglion (SG) nerves to induce hearing sensation (fig. 1a, b). Implants available today yield remarkable results for the vast majority of recipients with average postoperative word and sentence recognition approximating 60 and $70 \%$ correct, respectively, for unilaterally implanted recipients and 70 and $80 \%$ correct for bilateral recipients [Buss et al., 2008; Dorman et al., 2009; Gifford et al., 2008; Gifford et al., 2014a; Litovsky et al., 2006]. Despite this success, a significant number of users receive marginal benefit, and restoration to normal fidelity is rare even among the best performers. This is due, in part, to several well-known is-

\section{KARGER}

E-Mail karger@karger.com

www.karger.com/aud
(C) 2014 S. Karger AG, Basel

$1420-3030 / 14 / 0196-0400 \$ 39.50 / 0$
Jack $\mathrm{H}$. Noble, $\mathrm{PhD}$

Department of Electrical Engineering and Computer Science Vanderbilt University

2301 Vanderbilt Place, Box 1679B, Nashville, TN 37235 (USA)

E-Mail jack.h.noble@vanderbilt.edu

Website: www.vanderbilt.edu/CAOS/IGCIP 


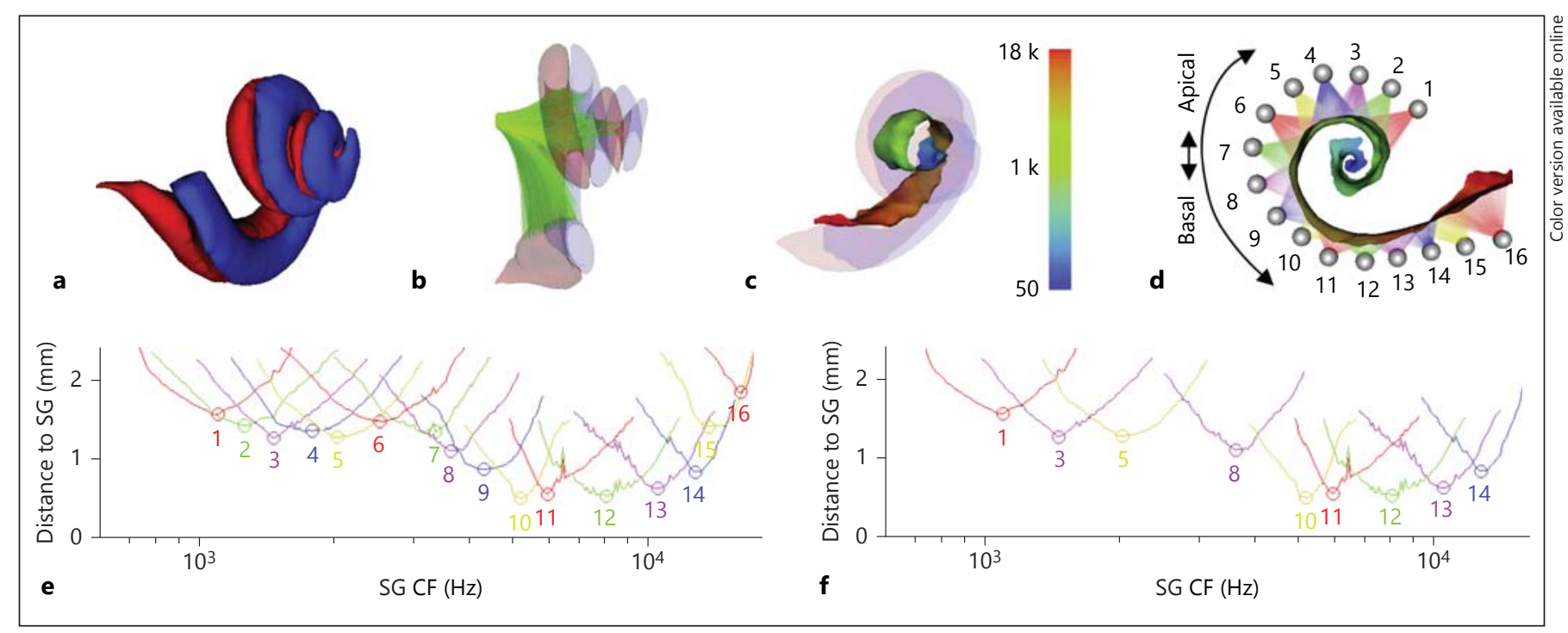

Fig. 1. Spatial analysis of an implanted subject. The scala tympani (red) and scala vestibuli (blue), the two principal cavities of the cochlea, are shown in a-c. $\mathbf{b}$ The auditory nerve fibers leading to the SG are shown in green. $\mathbf{c}$, d AR (the surface representing the interface between the nerves of the SG and the intracochlear cavities) is color-coded with the tonotopic place frequencies of the SG

sues with electrical stimulation that prevent CIs from accurately simulating natural acoustic hearing. Electrode interaction is an example of one such issue that, despite significant improvements made by advances in hardware and signal processing, remains challenging [Boex et al., 2003; Fu and Nogaki, 2005]. In natural hearing, a nerve pathway is activated when the characteristic frequency (CF) associated with that pathway is present in the incoming sound. Neural pathways are tonotopically ordered by decreasing CF along the length of the cochlear duct, and this finely tuned spatial organization is well known (fig. 1c) [Stakhovskaya et al., 2007]. CI electrode arrays are designed such that each electrode should stimulate nerve pathways corresponding to a predefined spectral bandwidth [Wilson and Dorman, 2008]. However, in surgery, the array is blindly threaded into the cochlea with its insertion path guided only by the walls of the spiralshaped intracochlear cavities. Since the final positions of the electrodes are generally unknown, the only option when programming has been to assume the electrodes are situated in the correct scala with a relatively uniform electrode-to-neuron interface across the array. Given this assumption, most implant recipients are programmed using a default frequency allocation table delivered across as many viable intracochlear electrodes as possible. Research has demonstrated, however, that many implanted in Hz. d The implanted electrodes of the CI, numbered 1-16, are also shown. An illustration of current spread from each electrode is rendered transparently with different colors between neighboring electrodes. Electrode distance-versus-frequency curves are plotted in e and f. (Colors refer to the online version only.)

arrays are not in the correct scala throughout the entire insertion depth [Finley et al., 2007; Holden et al., 2013; Skinner et al., 2007; Wanna et al., 2011], thus challenging the assumptions underlying a one-size-fits-all strategy.

Programming efficacy is sensitive to suboptimal electrode positioning [Rubenstein, 2004; Wilson and Dorman, 2008], which can lead to excessive spread of intracochlear electric current, and this is more commonly referred to as 'channel interaction' [Boex et al., 2003; Fu and Nogaki, 2005]. Thus, it follows that more effective implant programming could result from consideration of individualized electrode position. This is particularly true if we are able to reduce or eliminate the deleterious effects of channel interaction, which most notably includes poor spectral resolution. Spectral resolution is associated with peripheral filtering achieved via both the bank of overlapping auditory filters positioned along the basilar membrane as well as the tonotopic organization of SG neurons located within the modiolus. Impaired spectral resolution is known to result in significantly poorer speech recognition - particularly in background noise, as it results in a 'smearing' of the speech and noise spectra [e.g. Baer and Moore, 1994; Moore and Glasberg, 1993; Moore et al., 1995]. CI recipients are known to have poor spectral resolution [Henry and Turner, 2003, 2005; Litvak et al., 2007; Saoji and Eddins, 2007; Saoji et al., 2009; Spahr et 
al., 2011]. This is generally attributed to a number of factors, including that there is a discrete number of intracochlear electrodes, which limits the number of independent neural populations that can be stimulated [e.g. Friesen et al., 2001]; the population of surviving SG cells is unknown, and electric current spreads widely in the cochlea (i.e. channel interaction).

We have recently introduced image processing techniques that make it possible, for the first time, to estimate the position of implanted CI electrodes relative to the SG nerves they stimulate in computed tomography (CT) images [Noble et al., 2012]. Work from many other groups over the past decade has paved the way for in vivo electrode position analysis motivated by the importance of electrode location relative to the SG. For example, work has aimed to predict electrode insertion depth based on external cochlear dimensions such as basal turn diameter [Stakhovskaya et al., 2007] or the distance from the lateral wall to the round window [Escude et al., 2006]. Other groups have presented approaches for postoperatively imaging the location of implanted electrode arrays. For example, Aschendorff et al. [2005] and Verbist et al. [2005] optimized imaging protocols to permit better visualization of the electrodes in vivo. However, these techniques are still limited in that intracochlear structures are not well contrasted in CT, and the metallic electrodes further degrade tissue contrast due to image artifacts. Skinner et al. [2007] proposed an approach where intracochlear anatomy can be estimated in vivo in pre- and postimplantation CTs by rigidly aligning them with a high-resolution histological atlas of a specimen. The limitations of this approach are that it requires time and expertise to manually register the datasets, and it does not account for nonrigid variations in intracochlear anatomy. Our approach is semi-automatic, accurately estimates patient-specific intracochlear anatomy shape, and permits determining the location of the electrodes relative to intracochlear structures. This approach allows us to identify electrodes that provide overlapping stimulation patterns and to deactivate them from a patient's map [Noble et al., 2013]. In this article, we present results of experiments testing how this image-guided, patient-customized approach to stimulation, which we have termed image-guided cochlear implant programming (IGCIP), affects outcomes. Given that our current IGCIP approach is to deactivate electrodes thought to be causing overlapping electrical excitation, the overarching hypothesis driving this research is that our IGCIP strategy would reduce channel interaction, thereby improving spectral resolution. Should this strategy be effective at improving spectral resolution, we hypothesize that this would lead to corollary improvements in speech recognition, particularly in the presence of noise, and would further improve subjective perceptions of speech understanding abilities and overall sound quality.

The IGCIP methodology was originally proposed in Noble et al. [2013]. That publication also presents results from preliminary tests with 11 subjects. The principal contribution of this article is the reporting of results of an expanded clinical study with 68 new subjects. With this more substantial dataset, it is now possible to draw stronger conclusions from statistical analysis about how IGCIP strategies affect hearing performance.

\section{Materials and Methods}

In the following sections, we first present our approach for visualizing and analyzing the spatial relationship between the electrodes and the SG to facilitate the design of IGCIP strategies. Next, we introduce our proposed IGCIP strategy that uses this subjectspecific spatial information to reduce interaction of electrode stimulation patterns. Finally, we present details about our experimental design.

\section{Electrode Position Analysis}

We use the image processing techniques we have recently presented to detect the position of the implanted electrodes relative to the SG nerves [Noble et al., 2013]. These techniques are designed to detect electrode position using pre- and postimplantation CT scans. An example result of this process for one of our study subjects is shown in figure $1 \mathrm{~d}$. As can be seen, our software permits identifying the location of each contact in the array relative to the tonotopically mapped modiolus. Also shown are synthetically generated colored 'stimulation fields' that show how the neural stimulation patterns from each electrode might be shaped and how they overlap among neighboring electrodes. Analysis of the spatial relationship between the electrodes and the SG is necessary to extract programming-relevant information. Thus, to support the design of new image-guided programming strategies, we rely on a technique we developed in Noble et al. [2013] for visualizing programming-relevant spatial information that we call electrode distance-versus-frequency (DVF) curves. DVF curves are shown in figure 1e for one of our study subjects whose electrode positions are shown in figure 1d. In the plot, a DVF curve for each of the 16 electrodes is shown and colored similarly to the corresponding simulated current pattern shown in figure $1 \mathrm{~d}$. The $\mathrm{x}$-axis corresponds to CF of the SG in log-scale and the $y$-axis corresponds to the distance to the SG in millimeters. Each DVF curve shows the distance from the respective electrode to the closest regions of the SG, organized by $\mathrm{CF}$. Each curve takes on a roughly parabolic shape with a minimum corresponding to the electrode's closest SG nerves (shown with a circle in the plot) and with tails that increase in distance for adjacent nerve groups that are further from the electrode. Given the nature of the spread of electrical current through tissue, the shape of each electrode's excitation pattern on the SG is inversely related to its distance from the SG neural populations. 
This is supported by electrical modeling simulations [Whiten, 2007] as well as the pitch discrimination tests commonly performed clinically with CIs that show that electrodes that are closer to more apical SG regions generally create lower perceived pitches [Donaldson et al., 2005]. Thus, using the DVF curves, not only is it easy to infer the region of the SG that a specific electrode will best stimulate (e.g. the nerve pathways with CFs around 10 $\mathrm{kHz}$ are closest to electrode 13), but also it is easy to detect when two electrodes stimulate the same region. For instance, a substantial portion of the DVF curve for electrode 7 falls above the DVF curve for electrode 8 , which suggests that if both electrodes are active, they will both stimulate many of the same neural populations, and hence create channel interaction.

\section{Image-Guided Programming Strategy}

Since its introduction, continuous interleaved sampling (CIS) [Wilson et al., 1991] has been widely adopted, and all CI manufacturers today use CIS-based strategies [Rubenstein, 2004]. CIS uses nonsimultaneous, interleaved pulses to decrease cross-electrode electric field channel interactions; however, this is implemented without precise knowledge of the relative location of the neural pathways and the electrodes. By integrating spatial information provided by our image-processing techniques, we can extend this concept to decrease electrode interactions at the neural level, i.e. reduce the cross-electrode neural stimulation site overlap. In our experiments, the reprogramming strategy is straightforward. We deactivate electrodes that are likely to cause stimulation overlap. One approach to reduce competing stimulation without image guidance would be to drastically reduce the number of active electrodes, e.g. with only 1 active electrode, there would be no competition. However, the tradeoff with reducing the number of active electrodes is that this reduces the already very limited number of spectral channels, further compressing the frequency spectrum. Thus, blindly deactivating enough electrodes to ensure reduced competition risks deactivating potentially useful electrodes, i.e. those with stimulation regions that have little competition, and this would also result in suboptimal signal quality. With our approach, we assume that each electrode best stimulates SG sites that are closest to it (we call this the peak activation region), and we hypothesize that a better electrode configuration is one that consists of as many active electrodes as possible under the constraint that they all receive relatively little competition in their peak activation region. This approach allows for stimulation overlap at SG sites between electrodes, which is inevitable with a nontrivial number of electrodes, while ensuring that there is a subset of nerves that each electrode stimulates somewhat independently. Thus, we chose to keep active a maximal subset of electrodes that have DVF curves that do not substantially overlap around their minima. For the subject whose DVF curves are shown in figure 1e, we chose to keep active electrodes $1,3,5,8,10,11,12,13$, and 14, resulting in the DVF plot shown in figure 1f. As can be seen in the plot, each DVF curve with this reduced set of electrodes has a concave segment around its minimum that is closer to the SG than any other electrode, which indicates that more independent stimulation patterns are achieved than with the traditional all-electrodes-on strategy. Conveniently, this approach does not conflict with existing signal processing strategies, and thus reprogramming does not require major processing changes.

In our experiments, after identified electrodes are deactivated, the sound spectrum is remapped to the remaining active electrodes and the stimulation speed adjusted to account for the deactivated electrodes using the CI manufacturer's clinical software. The following section details our experimental design.

\section{Experimental Design}

Participants

Table 1 summarizes details about the study participants. Imageguided programming was completed for 72 ears in 68 postlingually deafened CI users (22 bilateral and 46 unilateral). Prior to this study, each of these subjects had undergone several iterations of traditional programming adjustments and was considered by an expert audiologist to have achieved a stable map and hence the best hearing performance possible using the traditional behavioral programming approach. The length of CI use among subjects ranged from 0.5 to 14.7 years with an average of 2.9 years. The right-hand columns of table 1 contain results that will be discussed below. Informed consent was obtained from each participant in accordance with the study protocols approved by the Vanderbilt Institutional Review Board.

\section{Experiment Summary}

For each participant, a battery of hearing and speech recognition tests was administered in up to three listening conditions: listening in the bilateral, best-aided condition; listening with the implanted ear being remapped alone, and, if the contralateral ear is also implanted, listening with the other implanted ear alone to serve as a control. Following baseline testing, the participant's CI was reprogrammed according to our image-guided programming strategy. So that performance with a new program could be measured in a semi-chronic condition, each subject returned for postadjustment retesting 3-6 weeks following the reprogramming. During this 3- to 6-week period, each subject was asked to live as they normally would; however, they were only provided with the new map so as to require compliance with full-time use of the experimental map. The difference between post- and pre-reprogramming hearing test results was used to quantify the benefit of our image-guided strategy. For bilateral recipients, we completed the reprogramming experiment on the poorer performing ear. Subjects 2, 4, and 37 had preimplantation CT scans on both ears and were able to participate in the study for both CIs. For these subjects, we subsequently performed the experiment on the contralateral CI.

The assessment of speech recognition was accomplished with the adult Minimum Speech Test Battery (MSTB) for adult CI recipients in the US [MSTB, 2011]. Estimates of spectral resolution were obtained using a spectral modulation detection (SMD) task, which is a non-speech-based hearing performance metric that provides a psychoacoustic estimate of spectral resolution, i.e. the ability of the auditory system to decompose a complex spectral stimulus into its individual frequency components [Drennan et al., 2010, 2014; Gifford et al., 2014b; Henry and Turner, 2003; Saoji et al., 2009].

Finally, to measure performance qualitatively, participants completed the Abbreviated Profile of Hearing Aid Benefit (APHAB [Cox and Alexander, 1995]) as well as the Speech, Spatial and Qualities of Hearing Scale (SSQ [Gatehouse and Noble, 2004]). All speech and non-speech stimuli were presented at a calibrated presentation level of $60 \mathrm{dBA}$ using a single loudspeaker presented at $0^{\circ}$ azimuth at a distance of $1 \mathrm{~m}$.

For participants with Advanced Bionics $(\mathrm{AB})$ implants, prior to deactivating the selected electrodes in the clinical software, we re- 
Table 1. Details of the study participants

\begin{tabular}{|c|c|c|c|c|c|c|c|c|c|c|c|}
\hline Experiment & Subject & $\begin{array}{l}\text { Bilateral } \\
\text { CIs }\end{array}$ & $\begin{array}{l}\text { Adjusted } \\
\text { ear }\end{array}$ & $\begin{array}{l}\text { Age, } \\
\text { years }\end{array}$ & $\begin{array}{l}\text { CI use, } \\
\text { years }\end{array}$ & $\begin{array}{l}\text { CI } \\
\text { brand }\end{array}$ & $\begin{array}{l}\text { Kept } \\
\text { map }\end{array}$ & MMSE & $\begin{array}{l}\text { Aided } \\
\text { SII }\end{array}$ & $\begin{array}{l}\text { Clinical } \\
\text { contacts }\end{array}$ & $\begin{array}{l}\text { Experimental } \\
\text { contacts }\end{array}$ \\
\hline 1 & 1 & no & $\mathrm{R}$ & 46 & 1.27 & $\mathrm{CO}$ & yes & & & 20 & 13 \\
\hline 3 & 2 & yes & $\mathrm{L}$ & 76 & 1.53 & $\mathrm{CO}$ & yes & & & 22 & 16 \\
\hline 4 & 3 & no & $\mathrm{R}$ & 69 & 2.14 & $\mathrm{CO}$ & yes & 30 & 23 & 20 & 19 \\
\hline 7 & 5 & no & $\mathrm{L}$ & 83 & 0.66 & $\mathrm{CO}$ & yes & 27 & 15 & 19 & 10 \\
\hline 10 & 8 & no & $\mathrm{L}$ & 70 & 3.03 & $\mathrm{CO}$ & yes & 28 & & 22 & 16 \\
\hline 13 & 11 & no & $\mathrm{R}$ & 86 & 1.27 & $\mathrm{CO}$ & no & 30 & & 22 & 15 \\
\hline 14 & 12 & yes & $\mathrm{L}$ & 55 & 3.43 & $\mathrm{CO}$ & yes & 30 & & 22 & 15 \\
\hline 15 & 13 & no & $\mathrm{R}$ & 53 & 2.45 & $\mathrm{CO}$ & yes & 30 & 25 & 22 & 11 \\
\hline 16 & 14 & yes & $\mathrm{R}$ & 62 & 7.58 & $\mathrm{CO}$ & yes & 30 & & 22 & 17 \\
\hline 22 & 20 & no & $\mathrm{L}$ & 69 & 0.67 & $\mathrm{CO}$ & yes & 29 & 27 & 21 & 14 \\
\hline 23 & 21 & no & $\mathrm{R}$ & 82 & 0.98 & $\mathrm{CO}$ & no & 30 & & 22 & 15 \\
\hline 26 & 24 & no & $\mathrm{R}$ & 78 & 0.9 & $\mathrm{CO}$ & no & & & 22 & 12 \\
\hline 31 & 28 & no & $\mathrm{R}$ & 68 & 1.32 & $\mathrm{CO}$ & yes & 29 & 42 & 22 & 15 \\
\hline 32 & 29 & no & $\mathrm{R}$ & 91 & 1.27 & $\mathrm{CO}$ & yes & 29 & 9 & 22 & 12 \\
\hline 34 & 31 & no & $\mathrm{L}$ & 84 & 2.84 & $\mathrm{CO}$ & yes & 23 & & 20 & 15 \\
\hline 35 & 32 & yes & $\mathrm{L}$ & 58 & 1.24 & $\mathrm{CO}$ & yes & 29 & & 17 & 6 \\
\hline 37 & 34 & no & $\mathrm{R}$ & 74 & 0.86 & $\mathrm{CO}$ & yes & 28 & 40 & 22 & 13 \\
\hline 39 & 36 & no & $\mathrm{R}$ & 69 & 1.35 & $\mathrm{CO}$ & yes & 27 & 30 & 22 & 17 \\
\hline 42 & 38 & no & $\mathrm{R}$ & 62 & 8.59 & $\mathrm{CO}$ & no & 29 & & 20 & 15 \\
\hline 44 & 40 & no & $\mathrm{L}$ & 83 & 1.53 & $\mathrm{CO}$ & yes & 30 & & 22 & 17 \\
\hline 45 & 41 & no & $\mathrm{R}$ & 79 & 2.13 & $\mathrm{CO}$ & yes & 26 & & 22 & 12 \\
\hline 60 & 56 & no & $\mathrm{R}$ & 66 & 1.13 & $\mathrm{CO}$ & yes & 29 & & 22 & 12 \\
\hline 62 & 58 & yes & $\mathrm{L}$ & 53 & 0.61 & $\mathrm{CO}$ & yes & 29 & & 20 & 18 \\
\hline 63 & 59 & no & $\mathrm{L}$ & 79 & 0.53 & $\mathrm{CO}$ & no & 30 & 24 & 21 & 15 \\
\hline 64 & 60 & yes & $\mathrm{R}$ & 78 & 11.91 & $\mathrm{CO}$ & yes & 26 & & 20 & 11 \\
\hline 67 & 63 & no & $\mathrm{L}$ & 60 & 1.32 & $\mathrm{CO}$ & no & 30 & 16 & 22 & 10 \\
\hline 68 & 64 & no & $\mathrm{R}$ & 55 & 0.81 & $\mathrm{CO}$ & no & 29 & 29 & 22 & 10 \\
\hline 69 & 65 & no & $\mathrm{R}$ & 77 & 1.83 & $\mathrm{CO}$ & yes & 28 & & 20 & 9 \\
\hline 70 & 66 & yes & $\mathrm{R}$ & 39 & 5.58 & $\mathrm{CO}$ & yes & 30 & & 20 & 11 \\
\hline 71 & 67 & no & $\mathrm{L}$ & 73 & 1.27 & $\mathrm{CO}$ & yes & 30 & 37 & 11 & 14 \\
\hline 72 & 68 & yes & $\mathrm{L}$ & 70 & 3.1 & $\mathrm{CO}$ & no & 26 & & 21 & 12 \\
\hline 28 & 26 & no & $\mathrm{L}$ & 43 & 0.75 & $\mathrm{ME}$ & yes & 30 & & 12 & 9 \\
\hline 29 & 27 & no & $\mathrm{R}$ & 66 & 0.67 & $\mathrm{ME}$ & no & 30 & & 12 & 10 \\
\hline 40 & 37 & yes & $\mathrm{L}$ & 59 & 2.53 & $\mathrm{ME}$ & yes & 30 & & 12 & 11 \\
\hline 41 & 37 & yes & $\mathrm{R}$ & 59 & 4.04 & $\mathrm{ME}$ & yes & & & 11 & 10 \\
\hline 46 & 42 & no & $\mathrm{R}$ & 64 & 0.86 & $\mathrm{ME}$ & yes & 28 & & 11 & 8 \\
\hline 47 & 43 & no & $\mathrm{R}$ & 76 & 11.2 & $\mathrm{ME}$ & yes & & & 11 & 9 \\
\hline 48 & 44 & no & $\mathrm{R}$ & 81 & 0.92 & $\mathrm{ME}$ & yes & 28 & & 11 & 9 \\
\hline 49 & 45 & no & $\mathrm{L}$ & 49 & 0.56 & $\mathrm{ME}$ & yes & 30 & & 12 & 9 \\
\hline
\end{tabular}


Table 1. (continued)

\begin{tabular}{|c|c|c|c|c|c|c|c|c|c|c|c|}
\hline Experiment & Subject & $\begin{array}{l}\text { Bilateral } \\
\text { CIs }\end{array}$ & $\begin{array}{l}\text { Adjusted } \\
\text { ear }\end{array}$ & $\begin{array}{l}\text { Age, } \\
\text { years }\end{array}$ & $\begin{array}{l}\text { CI use, } \\
\text { years }\end{array}$ & $\begin{array}{l}\text { CI } \\
\text { brand }\end{array}$ & $\begin{array}{l}\text { Kept } \\
\text { map }\end{array}$ & MMSE & $\begin{array}{l}\text { Aided } \\
\text { SII }\end{array}$ & $\begin{array}{l}\text { Clinical } \\
\text { contacts }\end{array}$ & $\begin{array}{l}\text { Experimental } \\
\text { contacts }\end{array}$ \\
\hline 50 & 46 & no & $\mathrm{R}$ & 64 & 1.4 & $\mathrm{ME}$ & yes & 29 & 11 & 11 & 8 \\
\hline 66 & 62 & yes & $\mathrm{L}$ & 64 & 6.43 & $\mathrm{ME}$ & yes & 30 & & 12 & 10 \\
\hline 5 & 4 & yes & $\mathrm{R}$ & 44 & 5.51 & $\mathrm{AB}$ & yes & 30 & & 16 & 11 \\
\hline 6 & 4 & yes & $\mathrm{L}$ & 44 & 5.51 & $\mathrm{AB}$ & yes & & & 16 & 14 \\
\hline 21 & 19 & no & $\mathrm{R}$ & 65 & 0.69 & $\mathrm{AB}$ & no & 28 & & 16 & 10 \\
\hline 24 & 22 & no & $\mathrm{R}$ & 68 & 5.8 & $\mathrm{AB}$ & yes & 30 & 20 & 16 & 12 \\
\hline 25 & 23 & no & $\mathrm{L}$ & 49 & 0.76 & $\mathrm{AB}$ & yes & 28 & & 15 & 7 \\
\hline 27 & 25 & no & $\mathrm{R}$ & 77 & 12.68 & $\mathrm{AB}$ & yes & 30 & & 16 & 12 \\
\hline 30 & 27 & yes & $\mathrm{R}$ & 66 & 0.51 & $\mathrm{AB}$ & yes & & & 16 & 9 \\
\hline 51 & 47 & no & $\mathrm{R}$ & 59 & 4.27 & $\mathrm{AB}$ & no & & 22 & 16 & 8 \\
\hline 61 & 57 & no & $\mathrm{L}$ & 54 & 0.82 & $\mathrm{AB}$ & no & 30 & & 16 & 11 \\
\hline
\end{tabular}

For each participant, the following characteristics are presented: whether (yes) or not (no) they have bilateral CIs, age in years, MMSE score, and aided SII score. For each experiment, the following factors are presented: the adjusted ear: left (L) or right (R); length of prior use of the adjusted CI in years; the manufacturer of the adjusted CI: AB, Cochlear (CO), or MED-EL (ME); whether the subject elected to keep the experimental map (yes) or not (no); the number of electrodes that were deactivated in the clinical map, and the number of electrodes that were deactivated in the experimental map.

moved Fidelity120 processing (i.e. current steering) to allow selective deactivation of electrodes without deleting a viable electrode in the 'pair'. For individuals making use of ClearVoice prior to study enrollment, removal of Fidelity120 required deactivation of ClearVoice as well. ClearVoice is described by $\mathrm{AB}$ as a signal or speech enhancement strategy. Though details of ClearVoice are guarded by proprietary restraint, it has been described as an algorithm designed to estimate the signal-to-noise ratio (SNR) level in each channel, and subsequently, for those channels in which noise and/ or poor SNR is identified, channel gain is reduced. We kept the stimulation strategy consistent with respect to paired or sequential stimulation. Further, pulse width was manipulated within $\pm 7 \mathrm{~ms}$ to keep channel stimulation rate consistent with the participant's clinical map. In some cases, this required switching from automatic pulse width in the SoundWave software to manually determined pulse width. We were careful to ensure that the resultant channel stimulation rate was within 400 pps of the participant's beginning rate with Fidelity120. Stimulation rates for all $A B$ recipients remained above 1,500 pps even after removing Fidelity120. For participants with MED-EL implants, we kept the participant's strategy consistent (high definition CIS or fine structure processing) and fixed the stimulation rate manually to that which was used in the patient's own map. All participants with Cochlear Corporation implants used an advanced combination encoder programming strategy. For these participants, after deactivating the selected electrodes, if the participant had 12 or fewer active electrodes in their map, we set the number of maxima equivalent to the number of active elec- trodes, thereby converting to a CIS program. For participants with more than 12 active electrodes in their experimental map, we kept the maxima consistent with what was used in the patient's own map which ranged from 8 to 12 maxima. Channel stimulation rate and pulse width were unaltered. For all implant recipients, regardless of implant manufacturer, manipulation of $\mathrm{M} / \mathrm{C}$ or $\mathrm{T}$ levels of individual electrodes was not performed. For patients reporting significantly 'softer' or less frequently 'louder' programs following selective electrode deactivation, we would globally increase or decrease $\mathrm{M} / \mathrm{C}$ levels to the participant's desired overall volume.

Study data were collected and managed using the REDCap (Research Electronic Data Capture) secure data managements tools hosted at Vanderbilt [Harris et al., 2009].

\section{Hearing Aid Verification}

The hearing aid (HA) settings for the contralateral ear of unilaterally implanted participants with residual acoustic hearing in the nonimplanted ear were verified prior to each test session. HAs were verified for all subjects using probe microphone measurements to NAL-NL2 [Dillon, 2006; Keidser et al., 2011] target audibility for $60-\mathrm{dB}$ sound pressure level speech. In cases where settings were undershooting NAL-NL2 target audibility, the participant's own HA was reprogrammed. In cases for which the participant's own HA was not adjustable due to either lack of reserve gain or incompatibility with NOAH programming software, a clinic stock HA was programmed and used for testing purposes. This occurred for 1 subject (No. 20) for which a Phonak Naida S V UP behind- 
the-ear HA was programmed, with SoundRecover deactivated, and affixed the participant's own fitted earmold. The aided speech intelligibility index (SII) values for $60-\mathrm{dB}$ sound pressure level speech and the unaided low-frequency pure tone average (125, 250 , and $500 \mathrm{~Hz}$ ), in $\mathrm{dB}$ hearing level, are provided in table 1 for the 19 bimodal participants in the current study.

Speech Recognition

Speech recognition was assessed as recommended by the revised MSTB [2011] for adult CI recipients. The MSTB outlines the administration of consonant-nucleus-consonant (CNC [Peterson and Lehiste, 1962]) monosyllabic words and AzBio sentences [Spahr et al., 2012] in quiet and noise. In addition to CNC words and AzBio sentences, we also assessed speech recognition in pseudoadaptive noise with the Bamford-Kowal-Bench Speech-in-Noise (BKB-SIN [Bench et al., 1979; Etymotic Research, 2005; Killion et al., 2004]) test. As compared to CNC and AzBio, which are scored in terms of percent correct, the BKB-SIN metric provides a score corresponding to the SNR at which the listener would achieve approximately $50 \%$ correct performance. This is reported as the SNR-50.

Participants scoring 50\% or higher for AzBio sentences in quiet were also tested at $+10 \mathrm{~dB}$ SNR using a continuous, multi-talker background noise. Similarly, participants scoring $50 \%$ or higher for AzBio sentences at $+10 \mathrm{~dB}$ SNR were also tested at $+5 \mathrm{~dB}$ SNR using the same continuous multi-talker babble. All speech tests were administered to each implanted ear independently as well as in the bilateral-aided condition whether that included bilateral implants $(\mathrm{CI}+\mathrm{CI})$ or bimodal hearing $(\mathrm{CI}+\mathrm{HA})$. For participants with bimodal hearing, the CI-only condition was assessed with the contralateral ear occluded via foam earplug.

\section{Assessment of Spectral Resolution: SMD}

Spectral resolution was assessed via SMD. The quick SMD task [Gifford et al., 2014b] used in the current study included a 3-inter$\mathrm{val}$, forced-choice procedure to contrast flat-spectrum noises with spectrally modulated noises. Spectral modulation was achieved by applying logarithmically spaced, sinusoidal modulation to the broadband carrier stimulus. The carrier stimulus had a bandwidth of $125-5,600 \mathrm{~Hz}$ [Gifford et al., 2014b]. SMD was assessed in the current study using a procedure based on a modified method of constant stimuli [Fechner, 1966; Gescheider, 1997]. There were 6 trials presented for each of the 5 modulation depths $(10,11,13,14$, and $16 \mathrm{~dB}$ ) and frequency [0.5 and 1.0 cycle/octave (cyc/oct)] for a total of 60 trials. Each trial was scored as correct or incorrect and spectral resolution is described as the overall percent correct score for the task $($ chance $=1 / 3$ ).

Perceived Hearing Handicap and Quality of Life

The APHAB provides a global estimate of the percentage of problems associated with listening in a variety of listening conditions and assesses aided benefit in 4 subscales including ease of communication, background noise, reverberation, and aversiveness. As such, lower scores on APHAB indicate fewer problems and better perceived benefit. The SSQ employs a visual analog scale which gauges hearing ability across listening domains including speech understanding in various listening conditions, spatial hearing associated with distance, movement and direction, and the overall quality of speech including clarity and naturalness of sound. Higher scores on this metric are correlated with better speech understanding, spatial hearing, and sound quality.

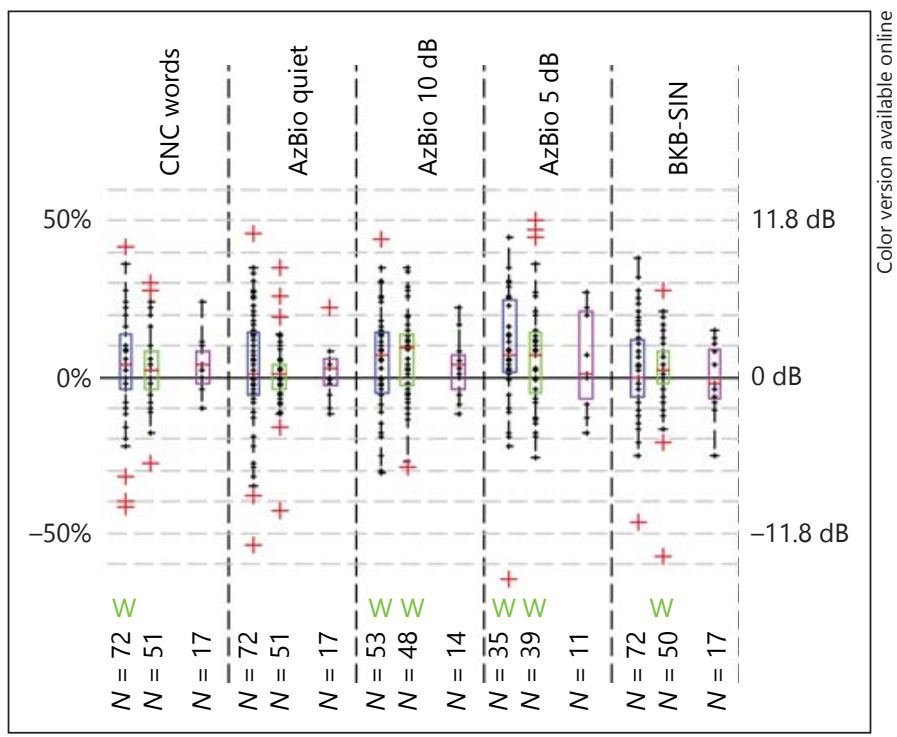

Fig. 2. Box plots of the raw benefit between post- and preadjustment condition testing of each hearing performance measure. Shown are the median (red line) and the range of the 75th to 25th percentile, i.e. $Q_{3}$ to $Q_{1}$ (box). Whiskers extend to data points that lie within the range $Q_{3}+1.5\left(Q_{3}-Q_{1}\right)$ to $Q_{1}-1.5\left(Q_{3}-Q_{1}\right)$. Outlier points that lie beyond the whiskers are shown as red pluses. Below each plot, a green ' $W$ ' indicates that the measure is statistically significant. Also shown is the dataset size $(N)$. For each measure, benefit is shown for the adjusted ear alone (blue, left box plot), bilateral (green, middle box plot), and control ear alone (magenta, right box plot) listening conditions. (Colors refer to the online version only.)

Screening for Cognitive Impairment

To enable detection of cognitive function-related effects, we administered the Mini-Mental State Examination (MMSE [Folstein et al., 1975]) screening tool at the time of study enrollment. The MMSE is a validated screening tool of cognitive function and includes tests of orientation, attention, memory, language, and visual-spatial skills. It is generally accepted that MMSE scores under 25 indicate impaired cognition.

\section{Results}

In figure 2, the box plots show the distributions across subjects of raw benefit computed as preadjustment scores subtracted from postadjustment scores. For each measure, benefit is shown for the adjusted ear alone (left box plot), bilateral (middle box plot), and control ear alone (right box plot) listening conditions. In each box plot, the short horizontal line indicates the median, the box indicates the 25th and 75th percentiles, red pluses indicate outliers, and the whiskers indicate the range of non-out- 
lier data. Below each box plot, the number of scores in the dataset $(N)$ is shown, and scores that are statistically significant as measured by the two-tailed Wilcoxon signed-rank test [Wilcoxon, 1945] at $\mathrm{p}<0.05$ are indicated with a green ' $W$ '. The dataset size, $N$, differs from plot to plot since test materials were not returned by some participants and not all measures were tested for every subject. The BKB-SIN measure is scored in terms of $\mathrm{dB}$, and units for benefit in $\mathrm{dB}$ for this measure are shown on the right. The remaining measures (CNC and AzBio) are scored in terms of percent correct, and these units are shown on the left. As seen in the figures, results of the pre- and postadjustment tests performed on the unadjusted control ear alone show, on average, little change. In contrast, the group average test results in the adjusted and bilateral conditions for several measures of speech in noise improved substantially, and improvements in several measures of hearing performance were statistically significant. Measures scored in percent correct are sensitive to range saturation effects when scores are closer to 0 or $100 \%$. When viewing raw benefit alone, range saturation effects can confound significant decline or improvement, especially in the bilateral listening condition where many participants already have relatively high scores with use of the contralateral ear. Thus, in figure 3, we show the same data for these measures in terms of percent benefit rather than raw benefit to account for ceiling and floor effects. Percent benefit is computed by normalizing the benefit or decline in each score with respect to the maximum possible benefit or decline. Thus, in this group, scores of $100 \%, 0 \%$, and $-100 \%$ represent a change from the preadjustment score to $100 \%$ correct, the same score, and $0 \%$ correct, respectively. We detected significant percent benefit for all quantitative measures of speech recognition in quiet and noise when tested in the bilateral condition. In contrast, we detected significant raw benefit as shown in figure 2 in the bilateral condition only for measures of speech in noise. This highlights the potential confound of benefit compression due to ceiling effects that occurs for the tests in quiet that are relatively easier and where raw pre-remapping scores are generally higher. Improvement in raw benefit for 3 of 5 measures when testing with the remapped ear alone are statistically significant, and improvement in percent benefit for 3 of 4 measures when testing in the same listening condition are also statistically significant.

Mean SMD scores are plotted in figure 4. SMD scores are shown, in percent correct, for each of the 5 spectral modulation depths (in $\mathrm{dB}$ ) as well as the mean across all depths for 0.5 and $1.0 \mathrm{cyc} /$ oct for the remapped ear alone.

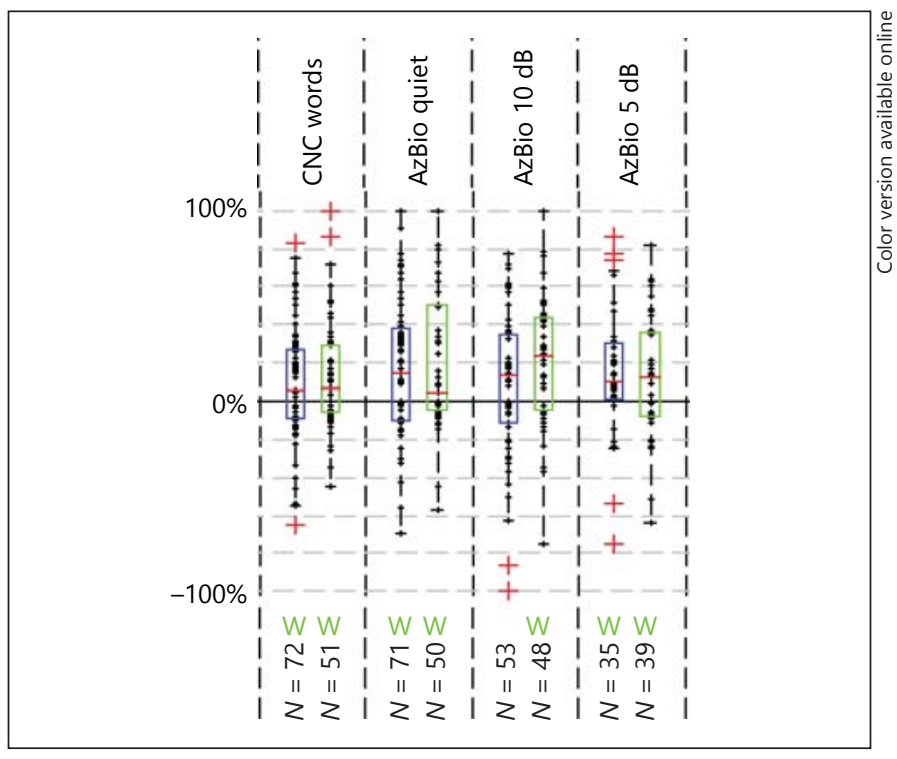

Fig. 3. Box plots of the percent benefit between post- and preadjustment condition testing of each hearing performance measure. Plot information is defined identically to figure 2 .

A two-way, repeated measures analysis of variance was completed with modulation depth and time point (prevs. postadjustment) as the independent variables and the SMD score as the dependent variable. For $0.5 \mathrm{cyc} / \mathrm{oct}$, statistical analysis revealed a significant effect of modulation depth $\left[\mathrm{F}_{(4,65)}=49.9, \mathrm{p}<0.001\right]$, a significant effect of time point $\left[\mathrm{F}_{(4,65)}=6.53, \mathrm{p}=0.013\right]$, and an interaction $\left[\mathrm{F}_{(4,65)}=\right.$ $3.9, \mathrm{p}=0.004]$. Post hoc testing using an all pairwise multiple comparison procedure (Holm-Sidak method) revealed a significant effect of time point for the two shallowest modulation depths of $10 \mathrm{~dB}(\mathrm{t}=2.5, \mathrm{p}=0.013)$ and $11 \mathrm{~dB}(\mathrm{t}=3.9, \mathrm{p}<0.001)$. For $1.0 \mathrm{cyc} / \mathrm{oct}$, statistical analysis revealed a significant effect of modulation depth $\left[\mathrm{F}_{(4,65)}=44.2, \mathrm{p}<0.001\right]$, no effect of time point $\left[\mathrm{F}_{(4,65)}=\right.$ $0.48, \mathrm{p}=0.49]$, and yet a significant interaction $\left[\mathrm{F}_{(4,65)}=\right.$ $2.6, \mathrm{p}=0.037]$. Post hoc testing using an all pairwise multiple comparison procedure (Holm-Sidak method) revealed a significant effect of time point for the shallowest modulation depth of $10 \mathrm{~dB}(\mathrm{t}=2.6, \mathrm{p}=0.01)$. Significant improvement in SMD is itself a substantial finding, as few developments in strategy in the past 20 years have been shown to significantly improve spectral resolution for CI recipients [Drennan et al., 2010]. More noteworthy is that the improvement in SMD was observed for the most shallow modulation depths $(10$ and $11 \mathrm{~dB})$ which are the most challenging conditions with respect to spectral envelope perception. 

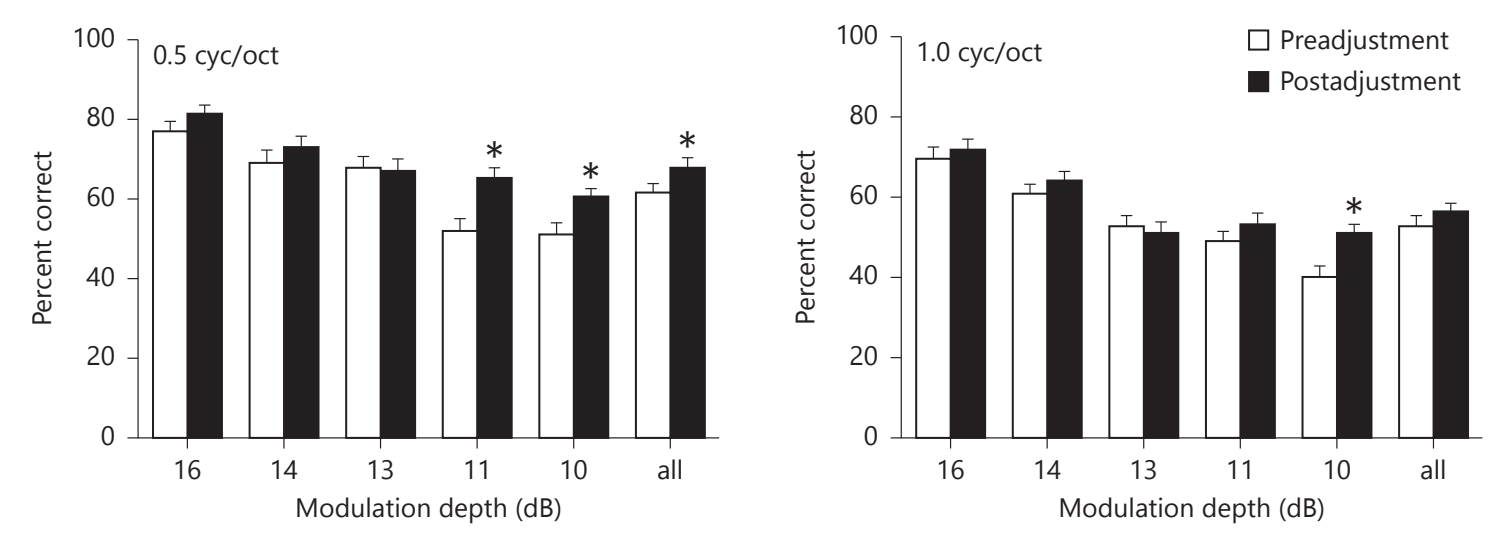

Fig. 4. Bar plots of mean SMD scores measured on the remapped ear alone. Scores are shown in percent correct for each of the 5 spectral modulation depths (in $\mathrm{dB}$ ) as well as the mean across all depths for 0.5 and 1.0 cyc/oct. A two-way, repeated measures anal- ysis of variance was completed with modulation depth and time point (pre- vs. postadjustment) as the independent variables and the SMD score as the dependent variable. Statistically significant results are indicated by asterisks.

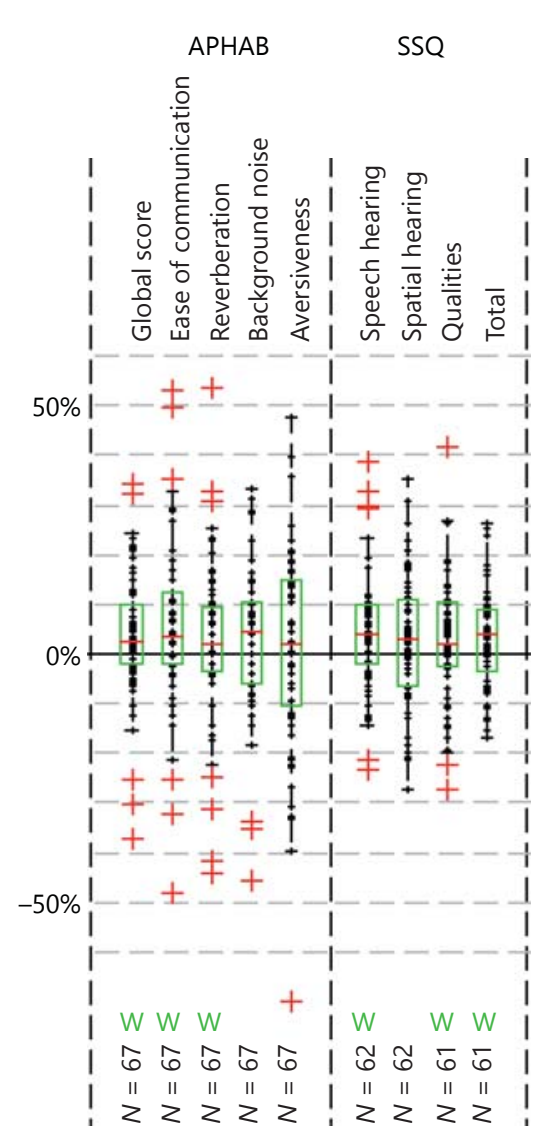

Fig. 5. Box plots of the qualitative benefit between post- and preadjustment condition measured by APHAB and SSQ. Plot information is defined identically to figure 2 .
Benefit in qualitative metrics (APHAB and SSQ) is shown in figure 5. Improvements in 5 of 7 individual components to these tests were statistically significant as were overall scores, indicating significant subjective preference to the experimental map on average.

Results for the adjusted ear of each subject are also shown as line plots in figure 6 and are color-coded by hearing test (Colors refer to the online version only). The left and right endpoints of each line plot indicate the pre- and post-remapping scores for the corresponding measure. Lines with positive slope indicate scores that improved from pre- to postadjustment. While some participants experienced decreases in hearing performance scores, the majority (64\%) of the line slopes are positive. Using critical difference tables generated for the CNC words [Thornton and Raffin, 1978] and AzBio sentences [Spahr et al., 2012] employing a binomial distribution statistic for individual speech perception metrics, we can detect significant differences between pre- and postadjustment scores on an individual basis for these measures. Further, for the BKB-SIN measure, Etymotic Research has published the critical difference value for adult CI recipients tested with 1 list pair being $4.4 \mathrm{~dB}$ based on the $95 \%$ confidence interval. Below the plots, scores that significantly improve and decline are indicated with green stars and blue circles. Measures that were not tested are indicated by a black ' $x$ '. There were statistically significant improvements at the individual level in 1 or more measures for 39 of 72 ears.

The right columns of table 1 indicate, for each experiment, whether the subject kept the experimental map and 


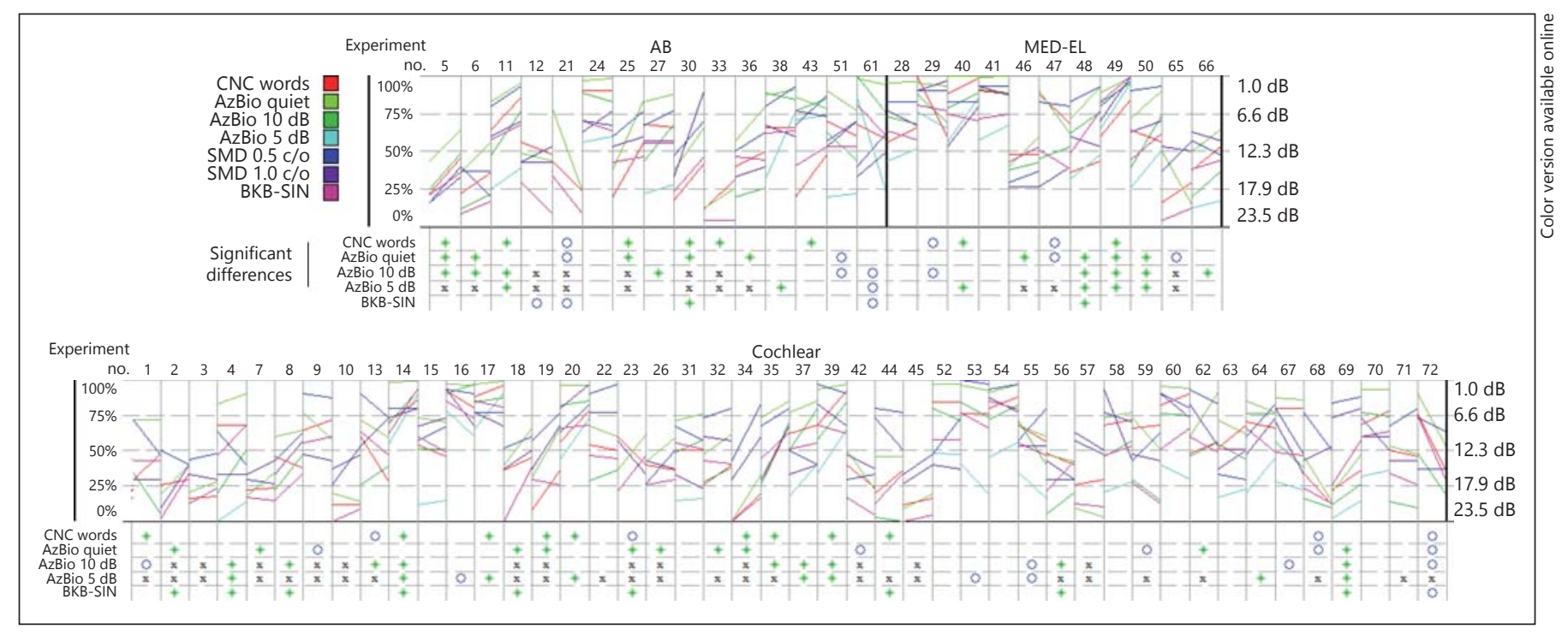

Fig. 6. Individual hearing performance results for the adjusted ear of each subject shown as line plots. The left and right ends of each line plot show pre- and postadjustment results for the indicated subject and hearing performance measure, respectively. Below the plots, scores that significantly improve and decline are indicated with green stars and blue circles. Measures that were not tested are indicated by a black ' $\mathrm{x}$ '. c/o = Cycle/octave. (Colors refer to the online version only.) the subject's MMSE and aided SII scores. The far right column shows the number of electrodes that were deactivated clinically, prior to this study, and the number of electrodes that our experimental techniques recommended for deactivation. As seen in table 1, participants requested changes or did not elect to keep their experimental maps for only 16 out of 72 ears $(22 \%)$. The remaining participants/ears (78\%) elected to keep the experimental maps with many strongly opposed to returning to their old maps. Many subjects who did not exhibit an individually significant benefit for speech or SMD testing reported improved sound quality and this is reflected here in the $78 \%$ rate of experimental map retention. Comments from individuals who participated in this study immediately after reprogramming and at the end of the study are included as online supplementary tables 1 and 2 (for all online suppl. material, see www.karger.com/doi/10.1159/000365273).

\section{Discussion}

The results of our image-guided CI programming tests show that our approach leads to significantly improved speech recognition, spectral resolution, and subjective hearing quality, and thus could improve hearing quality of life for many CI recipients. Statistically significant improvement was noted for the majority of the quantitative metrics tested. For the best-aided, bilateral condition, statistically significant raw improvement was noted for metrics that include noise, and statistically significant percent improvement was noted for all metrics. We believe this difference is due to the fact that improvements in metrics that are measured in quiet are masked in the raw scores due to range saturation effects for many of the subjects because they already had relatively high scores with the use of their better performing contralateral ear. The quality of overall change is best reflected in the APHAB and SSQ scores. Improvement in performance in background noise is especially significant considering that speech recognition in noise is one of the most common problems even among the best performing CI users [Fu and Noga$\mathrm{ki}, 2005]$. It is also of note that these results generally agree with the preliminary results reported in Noble et al. [2013], suggesting that results with our technique hold and are determined to be statistically significant when applied to a larger, relatively heterogeneous population.

Changes to hearing performance when electrodes are deactivated has been studied by other groups. For example, some groups have experimented with deactivating different numbers of electrodes in regular patterns and found little effect on average speech recognition as long as more than 4-8 electrodes are active [Friesen et al., 2001; Garnham et al., 2002]. Another study used electrode deactivation as a means to validate a psychophysical 
measure, although this technique did not lead to improvement in speech recognition over clinical maps [Garadat et al., 2012]. In another study, electrodes were deactivated based on an electrode pitch discrimination criterion leading to improvement in speech recognition for the majority of subjects in that 11 subject study [Zwolan et al., 1997]. This indicates it may be possible to use psychophysical measures to replicate our approach for selecting electrodes to deactivate. However, unlike most psychophysical metrics that require additional clinical testing and rely on subjective patient feedback, our image-guidance techniques do not require additional clinical time and are entirely objective.

Since the reprogramming strategy we use only requires deactivating electrodes, it is simple to integrate with existing sound processing strategies, such as CIS, using the existing clinical software provided by CI manufacturers. Typically, when changes to a program are made, quantitative and qualitative hearing scores tend to favor the original program [Tyler et al., 1986]. Thus, it is remarkable that the majority of the subjects in our experiments noted substantial improvement in sound quality immediately after reprogramming, and these improvements are reflected in our quantitative results. It is likely that long-term experience with the new program will result in further improvements in hearing performance. According to the National Institute on Deafness and Other Communication Disorders, over 200,000 people have received CIs as of 2010 [NIDCD, 2011]. We hypothesize that our electrode deac- tivation strategy could improve hearing in many of these CI users, thus improving their communication abilities and hearing-related quality of life, without requiring additional surgical procedures. Our results show that our personalized IGCIP approach to programming can improve spectral resolution and speech recognition in quiet and noise. However, the electrode deactivation strategy we present exploits only a small fraction of the programming-relevant information captured by our image processing and analysis techniques. Thus, we believe that the strategy tested in this study is just the first of many new and significant IGCIP stimulation strategies that will be developed now that analysis of the spatial relationship between electrodes and stimulation targets is possible.

\section{Acknowledgements}

This work was supported in part by grants R21DC012620, R01DC008408, and R01DC009404 from the National Institute on Deafness and Other Communication Disorders and UL1TR000445 from the National Center for Advancing Translational Sciences. The content is solely the responsibility of the authors and does not necessarily represent the official views of these institutes. This work was also supported by a Vanderbilt University Discovery Grant. The authors would like to acknowledge Mary S. Dietrich, $\mathrm{PhD}$, for advice on statistical analysis, Anthony J. Spahr, $\mathrm{PhD}$, and Chris Hetlinger, $\mathrm{PhD}$, for providing custom SMD testing software, as well as Linsey Sunderhaus, AuD, and Tim Davis, AuD, both for their assistance with data collection. Visit http://www.igcip.com to find our latest results and news.

\section{References}

Aschendorff A, Kubalek R, Turowski B, Zanella F, Hochmuth A, Schumacher M, Klenzner T, Laszig R: Quality control after cochlear implant surgery by means of rotational tomography. Otol Neurotol 2005;26:34-37.

Baer T, Moore BC: Effects of spectral smearing on the intelligibility of sentences in the presence of interfering speech. J Acoust Soc Am 1994; 95:2277-2280.

Bench J, Kowal A, Bamford J: The BKB (BamfordKowal-Bench) sentences lists for partiallyhearing children. Br J Audiol 1979;13:108112.

Boex C, de Balthasar C, Kos MI, Pelizzone M: Electrical field interactions in different cochlear implant systems. J Acoust Soc Am 2003;114:2049-2057.

- Buss E, Pillsbury HC, Buchman CA, Pillsbury $\mathrm{CH}$, Clark MS, Haynes DS, Labadie RF, Amberg S, Roland PS, Kruger P, Novak MA, Wirth JA, Black JM, Peters R, Lake J, Wackym PA, Firszt JB, Wilson BS, Lawson DT, Schatzer
R, D’Haese PS, Barco AL: Multicenter U.S. bilateral MED-EL cochlear implantation study: speech perception over the first year of use. Ear Hear 2008;29:20-32.

Cox RM, Alexander GC: The abbreviated profile of hearing aid benefit. Ear Hear 1995;16:176186.

Dillon H: What's new from NAL in hearing aid prescriptions? Hear J 2006;59:10-16.

Donaldson GS, Kreft HA, Litvak L: Place-pitch discrimination of single- versus dual-electrode stimuli by cochlear implant users. J Acoust Soc Am 2005;118:623-626.

Dorman MF, Yost W, Wilson BS, Gifford RH: Speech perception and sound localization by adults with bilateral cochlear implants. Semin Hear 2009;32:73-89.

Drennan WR, Won JH, Nie K, Jameyson E, Rubinstein JT: Sensitivity of psychophysical measures to signal processor modifications in cochlear implant users. Hear Res 2010;262: $1-8$.
Drennan WR, Anderson ES, Won JH, Rubenstein JT: Validation of a clinical assessment of spectral-ripple resolution for cochlear implant users. Ear Hear 2014;35:e92-e98.

Escudé B, James C, Deguine O, Cochard N, Eter E, Fraysse B: The size of the cochlea and predictions of insertion depth angles for cochlear implant electrodes. Audiol Neurootol 2006; 11(suppl 1):27-33.

Etymotic Research:Bamford-Kowal-Bench Speechin-Noise Test (version 1.03) (audio CD). 2005.

Fechner GT: Elements of Psychophysics (Engl. transl. by Adler HE); in Howes DH, Boring EC (eds): Henry Holt Editions in Psychology. Holt, Rinehart and Winston, Inc., 1966, p 47. (Originally published as Elemente der Psychophysik, 1860).

Finley CC, Holden TA, Holden LK, Whiting BR, Chole RA, Neely GJ, Hullar TE, Skinner MW: Role of electrode placement as a contributor to variability in cochlear implant outcomes. Otol Neurotol 2007;29:920-928. 
Folstein MF, Folstein SE, McHugh PR: MiniMental State. A practical method for grading the cognitive state of patients for the clinician. J Psychiatr Res 1975;12:189-198.

-Friesen LM, Shannon RV, Baskent D, Wang X: Speech recognition in noise as a function of the number of spectral channels: comparison of acoustic hearing and cochlear implants. J Acoust Soc Am 2001;110:11501163.

Fu QJ, Nogaki G: Noise susceptibility of cochlear implant users: the role of spectral resolution and smearing. J Assoc Res Otolaryngol 2005; 6:19-27.

-Garadat SN, Zwolan TA, Pfingst BE: Across-site patterns of modulation detection: relation to speech recognition. J Acoust Soc Am 2012; 131:4030-4041.

-Garnham C, O'Driscoll M, Ramsden R, Saeed S: Speech understanding in noise with a Med-El COMBI $40+$ cochlear implant using reduced channel sets. Ear Hear 2002;23:540-552.

-Gatehouse S, Noble W: The speech, spatial and qualities of hearing scale (SSQ). Int J Audiol 2004;43:85-99.

Gescheider G: The classical psychophysical methods; in Gescheider G: Psychophysics: The Fundamentals, ed 3. New York, Lawrence Erlbaum Associates, 1997, pp 45-72.

Gifford RH, Dorman MF, Sheffield SW, Teece K, Olund AP: Availability of binaural cues for bilateral implant recipients and bimodal listeners with and without preserved hearing in the implanted ear. Audiol Neurotol 2014a;19:57-71.

-Gifford RH, Hedley-Williams A, Spahr AJ: Clinical assessment of spectral modulation detection for adult cochlear implant recipients: a non-language based measure of performance outcomes. Audiol Neurotol 2014b;53:159164.

Gifford RH, Shallop JK, Peterson AM: Speech recognition materials and ceiling effects: considerations for cochlear implant programs. Audiol Neurotol 2008;13:193-205.

-Harris PA, Taylor R, Thielke R, Payne J, Gonzalez N, Conde JG: Research electronic data capture (REDCap) - a metadata-driven methodology and workflow process for providing translational research informatics support. J Biomed Inform 2009;42:377-381.

-Henry BA, Turner CW: The resolution of complex spectral patterns by cochlear implant and normal-hearing listeners. J Acoust Soc Am 2003; 113:2861-2873.

-Henry BA, Turner CW, Behrens A: Spectral peak resolution and speech recognition in quiet: normal hearing, hearing impaired, and cochlear implant listeners. J Acoust Soc Am 2005;118:1111-1121.
Holden LK, Finley CC, Firszt JB, Holden TA, Brenner C, Potts LG, Gotter BD, Vanderhoof SS, Mispagel K, Heydebrand G, Skinner MW: Factors affecting open-set word recognition in adults with cochlear implants. Ear Hear 2013;34:342-360.

Keidser G, Dillon H, Flax M, Ching TY, Brewer S: The NAL-NL2 prescription procedure. Audiol Res 2011;1:88-90.

-Killion MC, Niquette PA, Gudmundsen GI, Revit LJ, Banerjee S: Development of a quick speech-in-noise test for measuring signal-tonoise ratio loss in normal-hearing and hearing-impaired listeners. J Acoust Soc Am 2004; 116:2395-2405.

Litovsky RY, Parkinson A, Arcaroli J, Sammeth C: Simultaneous bilateral cochlear implantation in adults: a multicenter clinical study. Ear Hear 2006;27:714-730.

Litvak LM, Spahr AJ, Saoji A, Fridman GY: Relationship between perception of spectral ripple and speech recognition in cochlear implant and vocoder listeners. J Acoust Soc Am 2007; 122:982-991.

Moore BCJ, Glasberg BR: Simulation of the effects of loudness recruitment and threshold elevation on the intelligibility of speech in quiet and in a background of speech. J Acoust Soc Am 1993;94:2050-2062.

Moore BC, Glasberg BR, Vickers DA: Simulation of the effects of loudness recruitment on the intelligibility of speech in noise. Br J Audiol 1995;29:131-143.

MSTB: The New Minimum Speech Test Battery. http://www.auditorypotential.com/MSTB_ Nav.html, 2011.

NIDCD (National Institute on Deafness and Other Communication Disorders): Cochlear Implants. No. 11-4798, 2011.

- Noble JH, Gifford RH, Labadie RF, Dawant BM: Statistical shape model segmentation and frequency mapping of cochlear implant stimulation targets in CT; in Ayache $\mathrm{N}$ et al (eds): Medical Image Computing and ComputerAssisted Intervention - MICCAI 2012, Part II. Lecture Notes in Computer Science 7511. Berlin, Springer, 2012, pp 421-428.

Noble JH, Labadie RF, Gifford RH, Dawant BM: Image-guidance enables new methods for customizing cochlear implant stimulation strategies. IEEE Trans Neural Syst Rehabil Eng 2013;21:820-829.

Peterson GE, Lehiste I: Revised CNC lists for auditory tests. J Speech Hear Dis 1962;27:62-70.

Rubenstein JT: How cochlear implants encode speech. Curr Opin Otolaryngol Head Neck Surg 2004; 12:444-448.

-Saoji AA, Eddins DA: Spectral modulation masking patterns reveal tuning to spectral envelope frequency. J Acoust Soc Am 2007;122:10041013.
Saoji AA, Litvak LM, Spahr AJ, Eddins DA: Spectral modulation detection and vowel and consonant identifications in cochlear implant listeners. J Acoust Soc Am 2009;126:955-958.

- Skinner MW, Holden TA, Whiting BR, Voie AH, Brundsen B, Neely GJ, Saxon EA, Hullar TE, Finley CC: In vivo estimates of the position of advanced bionics electrode arrays in the human cochlea. Ann Otol Rhinol Laryngol Suppl 2007;197:2-24.

-Spahr AJ, Dorman MF, Litvak LM, Van Wie S, Gifford RH, Loizou PC, Loiselle LM, Oakes T, Cook S: Development and validation of the AzBio sentence lists. Ear Hear 2012;33:112117.

Spahr AJ, Saoji A, Litvak LM, Dorman MF: Spectral cues for understanding speech in quiet and in noise. Cochlear Implants Int 2011; 12:S66-S69.

-Stakhovskaya O, Spridhar D, Bonham BH, Leake PA: Frequency map for the human cochlear spiral ganglion: implications for cochlear implants. J Assoc Res Otol 2007;8:220-233.

Thornton AR, Raffin MJ: Speech-discrimination scores modeled as a binomial variable. J Speech Hear Res 1978;21:507-518.

Tyler RS, Preece JP, Lansing CR, Otto SR, Gantz BJ: Previous experience as a confounding factor in comparing cochlear-implant processing schemes. J Speech Hear Res 1986;29:282287.

Verbist BM, Frijns JHM, Geleijns J, van Buchem MA: Multisection CT as a valuable tool in the postoperative assessment of cochlear implant patients. AJNR Am J Neuroradiol 2005;26: 424-429.

-Wanna GB, Noble JH, McRacken TR, Dawant BM, Dietrich MS, Watkins LD, Schuman TA, Labadie RF: Assessment of electrode placement and audiologic outcomes in bilateral cochlear implantation. Otol Neurotol 2011;32: 428-432.

Whiten D: Electro-Anatomical Models of the Cochlear Implant; PhD thesis, Massachusetts Institute of Technology Library, 2007.

Wilcoxon F: Individual comparisons by ranking methods. Biometrics Bull 1945;1:80-83.

Wilson BS, Dorman MF: Cochlear implants: current designs and future possibilities. J Rehab Res Dev 2008;45:695-730.

-Wilson BS, Finley CC, Lawson DT, Wolford RD, Eddington DK, Rabinowitz WM: Better speech recognition with cochlear implants. Nature 1991;352:236-238.

Zwolan TA, Collins LM, Wakefiled GH: Electrode discrimination and speech recognition in postlingually deafened adult cochlear implant subjects. J Acoust Soc Am 1997;102: 3673-3685.
Image-Guided Cochlear Implant

Programming
Audiol Neurotol 2014;19:400-411 DOI: $10.1159 / 000365273$ 